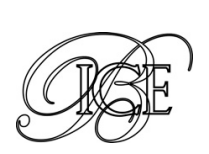

Judith Arnal Martinez*

\title{
APOYO FINANCIERO A LOS ESTADOS MIEMBROS DE LA ZONA EURO ANTE LA CRISIS DE DEUDA SOBERANA \\ El Mecanismo Europeo de Estabilidad
}

El estallido de la crisis de deuda soberana de la zona euro, que inicialmente afectó a Grecia, puso de manifiesto la falta de un entramado institucional que permitiera prestar asistencia financiera a aquellos Estados miembros que la requirieran. Tras la solución ad hoc de prestar asistencia financiera bilateral a Grecia a través de la Greek Loan Facility, las autoridades europeas crearon el Mecanismo Europeo de Estabilidad Financiera y el Fondo Europeo de Estabilización Financiera. No obstante, el Consejo Europeo de diciembre de 2010 acordó la necesidad de que los Estados miembros de la zona euro se dotaran de un mecanismo permanente de estabilidad. Fruto de este compromiso, en octubre de 2012 se inauguró el Mecanismo Europeo de Estabilidad, organización intergubernamental cuyo funcionamiento se describe en este artículo.

Palabras clave: recapitalización bancaria, programa de ajuste macroeconómico, asistencia financiera. Clasificación JEL: F33, F34, F36, H12.

\section{Introducción}

Cuando la crisis de deuda soberana en la zona euro se inició a finales de 2009, ni la Unión Europea ni los Estados miembros de la moneda única contaban con mecanismos de apoyo institucional que permitieran prestar asistencia financiera a aquellos países que la necesitaran. Ante esta ausencia de desarrollo institucional, los Estados miembros de la zona euro decidieron asistir a Grecia mediante la concesión, en abril de 2010, de una serie de préstamos bilaterales, conocidos bajo el nombre de Greek Loan Facility.

Sin embargo, la solución bilateral aplicada al caso griego no se consideró satisfactoria para posibles casos en el futuro, por lo que se inició un

\footnotetext{
* Técnico Comercial y Economista del Estado.

Versión de octubre de 2015.
}

proceso en el seno de la Unión Europea y la zona euro para crear una red de seguridad financiera.

A nivel de la Unión Europea, esta red se concretó en la aprobación del Reglamento por el que se creaba el Mecanismo Europeo de Estabilidad Financiera $^{1}$ (EFSM, por sus siglas en inglés). El EFSM es un mecanismo capaz de prestar asistencia financiera a cualquier Estado miembro de la Unión Europea ${ }^{2}$ con fondos captados por la Comisión Europea en $\triangleright$

\footnotetext{
1 Reglamento (UE) $n^{\circ}$ 407/2010 por el que se establece un Mecanismo Europeo de Estabilización Financiera, recientemente enmendado por el Reglamento (UE) $n^{\circ}$ 2015/1360, del Consejo de 4 de agosto de 2015.

2 Como consecuencia de la modificación del Reglamento en agosto de 2015, cualquier asistencia financiera que el EFSM conceda a un Estado miembro de la zona euro deberá contar con una garantía que asegure que los Estados miembros, cuya moneda no es el euro, sean inmediata e íntegramente compensados por la responsabilidad en que pudiesen incurrir si el Estado miembro beneficiario no reembolsa la ayuda financiera con arreglo a las condiciones de la misma.
} 
los mercados financieros. Cuenta con la garantía del Presupuesto de la Unión Europea. EI EFSM ha sido empleado en tres ocasiones: para el programa de asistencia financiera irlandés, por importe de 22.500 millones de euros; para el programa de asistencia financiera portugués, por importe de 24.300 millones de euros; y finalmente, para la financiación puente concedida a Grecia en julio de 2015, por importe de 7.160 millones de euros.

A nivel de la zona euro, se creó la Facilidad Europea de Estabilización Financiera (EFSF, por sus siglas en inglés), sociedad constituida bajo derecho privado luxemburgués el 7 de junio de 2010 con el objeto de preservar la estabilidad financiera de la zona euro, proporcionando asistencia financiera temporal a los Estados miembros que la necesitaran. Esta sociedad cuenta con las garantías concedidas por los Estados miembros de la zona euro, que respaldan sus emisiones en los mercados financieros. Aunque el importe máximo inicial de las garantías era de 440.000 millones de euros, el 24 de junio de 2010, los Jefes de Estado y de Gobierno de la zona euro decidieron incrementar el importe de las garantías hasta los 780.000 millones de euros, lo que otorgaba una capacidad máxima de préstamo efectiva al EFSF de 440.000 millones de euros.

El EFSF, que desde el 1 de julio de 2013 no puede conceder nuevos programas de asistencia financiera, ha intervenido en la concesión de tres programas diferentes: el programa irlandés, concluido en diciembre de 2013, por un importe de 17.700 millones de euros; el programa portugués, concluido en mayo de 2014, por un importe de 26.000 millones de euros; $y$ finalmente, el segundo programa griego, concluido en junio de 2015 tras dos extensiones, por un importe de 130.900 millones de euros.

A pesar del establecimiento de estos dos mecanismos, el Consejo Europeo de 17 de diciembre de 2010, en línea con sus propias conclusiones de 28 y 29 de octubre de 2010, estableció la necesidad de que los Estados miembros de la zona euro crearan un mecanismo permanente de estabilidad, que asumiera las tareas desempeñadas por el EFSF y el EFSM.
Con vistas a dar cumplimiento a su propio mandato, el 25 de marzo de 2011, el propio Consejo Europeo modificó el artículo 136 del Tratado de Funcionamiento de la Unión Europea (TFUE) ${ }^{3}$, permitiendo la creación de un mecanismo de estabilidad para el conjunto de la zona euro. De este modo, se gestó el nacimiento del Mecanismo Europeo de Estabilidad (ESM, por sus siglas en inglés).

EI ESM es una organización intergubernamental sometida a Derecho Internacional Público e inaugurada el 8 de octubre de 2012 tras la entrada en vigor, el 27 de septiembre de ese mismo año, del Tratado del ESM.

El objetivo fundacional del ESM es actuar como mecanismo permanente de resolución de crisis en los Estados miembros de la zona euro, proporcionando asistencia financiera a través de un conjunto de instrumentos.

Todos los Estados miembros de la zona euro serán miembros del ESM. Como consecuencia de la adhesión a la zona euro, todo Estado miembro deberá convertirse en integrante pleno del ESM. Así, aunque el ESM inició su andadura con 17 Estados miembros, las adhesiones de Letonia y Lituania a la zona euro en 2014 y 2015, respectivamente, han elevado el número de partícipes en el capital del ESM a 19 Estados miembros.

El propósito de este artículo es dar a conocer las características más relevantes de esta nueva institución financiera internacional, cuyo papel ha sido clave en el mantenimiento del proyecto de la moneda única. Para ello, en primer lugar, se hará referencia a la estructura organizativa del ESM, que gira en torno a un conjunto de consejos y comités que marcan su funcionamiento. En segundo lugar, se hará referencia al grupo de normas por las que ha de regirse el ESM, que como se verá, cuentan con un claro orden jerárquico que evita cualquier posible conflicto normativo. En tercer lugar, se $\triangleright$

\footnotetext{
3 Concretamente, se añadió el siguiente párrafo al artículo 136 TFUE: «Los Estados miembros cuya moneda es el euro podrán establecer un mecanismo de estabilidad que se activará cuando sea indispensable para salvaguardar la estabilidad de la zona euro en su conjunto. La concesión de toda ayuda financiera necesaria con arreglo al mecanismo se supeditará a condiciones estrictas».
} 
tratará la estructura financiera del ESM, que cuenta con un capital total superior a 700.000 millones de euros y un capital desembolsado de más de 80.000 millones de euros, que se invierten por la institución en activos de alta calidad y le permiten el apalancamiento en los mercados de capitales. En cuarto lugar, se explicará el procedimiento que se sigue para la concesión de un programa de asistencia financiera por el ESM. Finalmente, se dará una visión de los distintos instrumentos con que el ESM cuenta en la actualidad para prestar asistencia financiera.

\section{Estructura organizativa del ESM}

En cuanto a su estructura, el ESM está formado por un Consejo de Gobernadores (BoG, por sus siglas en inglés), un Consejo de Administración (BoD, por sus siglas en inglés), dos Comités dependientes del $\mathrm{BoD}$, un Consejo de Auditoría (BoA, por sus siglas en inglés), un Tribunal Administrativo propio de la institución (ESMAT, por sus siglas en inglés), un Director Ejecutivo (MD, por sus siglas en inglés), una Junta Directiva (MB, por sus siglas en inglés) y demás personal propio de la institución.

El BoG está formado por un gobernador y un gobernador suplente de cada Estado miembro ${ }^{4}$. Los gobernadores son los Ministros de Finanzas de los Estados miembros de la zona euro. El BoG puede decidir estar presidido bien por el Presidente del Eurogrupo, bien por uno de sus miembros durante un período de dos años ${ }^{5}$. El Comisario Europeo de Asuntos Económicos y Monetarios y el Presidente del Banco Central Europeo pueden asistir a las reuniones del BoG como miembros observadores. Asimismo, aquellos Estados miembros de la Unión Europea cuya moneda no es el euro, pero que participen sobre una base ad hoc, paralelamente al

\footnotetext{
4 En el momento de redactar este artículo, el gobernador por España es el Ministro de Economía y Competitividad, Luis de Guindos Jurado, y la gobernadora suplente la Secretaria General del Tesoro y Política Financiera, Rosa María Sánchez-Yebra Alonso.

5 El momento de redactar este artículo, el Presidente del BoG es el Presidente del Eurogrupo, el holandés Jeroen Dijsselbloem.
}

ESM, en una operación de apoyo a la estabilidad de un Estado miembro de la zona euro, podrán participar en el BoG en calidad de observadores.

El BoG adopta sus decisiones por unanimidad o por mayoría cualificada. Las decisiones de mayor trascendencia, como la concesión de asistencia financiera a un Estado miembro, la elección de los instrumentos, las condiciones y términos de dicha asistencia, las llamadas de capital exigible, las modificaciones en el capital total o los cambios en la capacidad máxima de préstamo, se toman por unanimidad. Otro tipo de decisiones, como el establecimiento de los detalles concretos de los términos de acceso de un Estado miembro al ESM, el nombramiento del MD o la aprobación de las cuentas anuales del ESM se adoptan por mayoría cualificada, que equivale al 80 por 100 de los votos expresados, calculados de forma ponderada en función de la contribución de cada Estado miembro al capital.

A pesar de que la decisión de conceder una asistencia financiera ha de adoptarse por el BoG por unanimidad, podría llegar a aplicarse el procedimiento de votación de urgencia si la Comisión Europea y el Banco Central Europeo concluyeran que la no adopción de forma urgente de la decisión de concesión de la asistencia financiera amenazara la sostenibilidad económica y financiera de la zona euro. La adopción de una decisión bajo el procedimiento de urgencia requiere una mayoría cualificada del 85 por 100 de los votos expresados.

El BoD está formado por un administrador y un administrador suplente de cada Estado miembro, designados por el gobernador ${ }^{6}$. El BoD está presidido por el MD. El Comisario Europeo de Asuntos Económicos y Monetarios y el Presidente del BCE pueden nombrar a un responsable de sus respectivas instituciones, que actúe a modo de observador. Asimismo, aquellos Estados miembros de la Unión Europea cuya moneda no es el euro, pero $\triangle$

\footnotetext{
6 En el momento de redactar este artículo, la administradora por España es la Secretaria General del Tesoro y Política Financiera Rosa María Sánchez-Yebra Alonso y la administradora suplente es la Subdirectora General de Asuntos Económicos y Financieros de la UE, Carla Díaz Álvarez de Toledo.
} 
que participen sobre una base ad hoc, paralelamente al ESM, en una operación de apoyo a la estabilidad de un Estado miembro de la zona euro, podrán participar también en el BoD en calidad de observadores.

El BoD adopta sus decisiones por mayoría cualificada, salvo cuando se trata de decisiones delegadas por el BoG, en cuyo caso se aplicarán las reglas de votación del propio BoG.

Existen dos comités que dependen del BoD: el Comité de Riesgos (BRC, por sus siglas en inglés) y el Comité de Compensaciones (BCC, por sus siglas en inglés). Ambos comités están compuestos por cinco miembros ${ }^{7}$. La labor fundamental del BRC consiste en asesorar al BoD acerca del apetito actual y futuro por el riesgo del ESM y controlar y revisar, por mandato del $\mathrm{BoD}$, el marco general de gestión de riesgos del ESM. Por su parte, el BCC tiene como misión principal asesorar al $\mathrm{BoD}$ sobre el marco y los principios de las compensaciones del personal del ESM.

El BoA es un cuerpo independiente formado por cinco miembros nombrados por el BoG ${ }^{8}$. El BoA está encargado de supervisar las cuentas del ESM y de controlar los procesos y resultados de las auditorías internas y externas de la institución.

EI ESMAT fue establecido en octubre de 2013. Es un órgano independiente, compuesto por cinco miembros $^{9}$ elegidos por un comité ${ }^{10}$. Su función principal consiste en resolver litigios en materia de personal, en los términos establecidos en su Estatuto, aprobado por el BoD.

El MD es nombrado por el BoG de entre candidatos que posean la nacionalidad de un miembro

\footnotetext{
7 En el momento de redactar este artículo, los representantes en el BRC son: Austria, Holanda, Italia (ostenta la presidencia del Comité), Portugal y República Eslovaca, y los representantes en el BCC Alemania, España, Irlanda, Luxemburgo y Malta (ostenta la presidencia del Comité).

8 Dos de los miembros del BoA son propuestos por el Presidente del BoG, otros dos por las máximas instituciones de auditoría de dos Estados miembros del ESM y el miembro restante por la Corte Europea de Auditores.

9 En el momento de redactar este artículo, los miembros del ESMAT son: Virginia Melgar (Presidente), Haris Tagaras (Vicepresidente), Julian Currall, Celia Goldman y Gerhard Ullrich.

10 El Comité está presidido por una persona escogida por el Presidente del BoG e integrado por el MD, el Consejero General, el Secretario General y un representante del personal del ESM.
}

del ESM, una experiencia internacional adecuada y un elevado nivel de competencia en el ámbito económico y financiero. El mandato del MD tiene una duración de cinco años, pudiendo renovarse una vez. Presidirá las reuniones del $\mathrm{BoD}$, participará en las reuniones del BoG, será el jefe del personal del ESM y el representante legal de la institución.

La MB tiene como misión principal asesorar al $\mathrm{MD}$ en su toma de decisiones cotidianas. Está compuesto por el MD y otros cinco miembros adicionales ${ }^{11}$.

En la actualidad, el ESM cuenta con un personal propio que ronda las 140 personas. Este personal es compartido con EFSF. Se trata de una plantilla relativamente reducida para las importantes funciones que la institución tiene atribuidas, lo que contribuye a reforzar el funcionamiento eficiente de la institución.

\section{Normativa reguladora del ESM}

EI ESM está gobernado por un conjunto de normas, que siguen una jerarquía determinada.

EI Tratado del ESM, que entró en vigor el 27 de septiembre de 2012, se encuentra en la cima de esta jerarquía normativa.

A continuación se sitúan los Estatutos del ESM, normativa de desarrollo del Tratado del ESM.

Las Reglas de Procedimiento se sitúan en el siguiente escalón normativo y regulan las normas de funcionamiento del BoG y del BoD del ESM.

Las políticas y guías del BoD continúan con esta pirámide normativa, regulando aspectos como las políticas de precios, inversión y financiación del ESM o las características específicas de las distintas modalidades de asistencia financiera.

La documentación legal de la asistencia financiera seguiría en rango legal a las normas anteriormente citadas. La documentación legal de la asistencia $\triangleright$

\footnotetext{
11 En el momento de redactar este artículo, estos miembros son: Kalin Anev Janse (Secretario General del ESM), Françoise Blondeel (responsable del Middle y Back Office del ESM), Christophe Frankel (ViceMD del ESM y Director de Finanzas del ESM), Ralf Jansen (Secretario General del ESM) y Rolf Strauch (responsable de Economía, Estrategia y Banca del ESM).
} 


\begin{tabular}{|c|c|c|c|}
\hline Estado miembro & Clave de contribución (\%) & $\begin{array}{c}\text { Capital total } \\
\text { (en millones de euros) }\end{array}$ & $\begin{array}{l}\text { Capital desembolsado } \\
\text { (en millones de euros) }\end{array}$ \\
\hline Alemania & 26,9616 & 190.020 & 21.720 \\
\hline Austria & 2,7644 & 19.480 & 2.230 \\
\hline 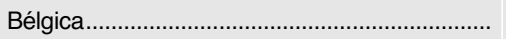 & 3,4534 & 24.340 & 2.780 \\
\hline 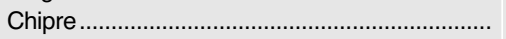 & 0,1949 & 1.370 & 160 \\
\hline 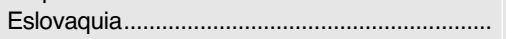 & 0,8184 & 5.770 & 660 \\
\hline Eslovenia & 0,4247 & 2.990 & 340 \\
\hline España & 11,8227 & 83.330 & 9.520 \\
\hline 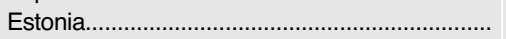 & 0,1847 & 1.300 & 150 \\
\hline Finlandia & 1,7852 & 12.580 & 1.440 \\
\hline 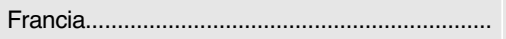 & 20,2471 & 142.700 & 16.310 \\
\hline Grecia & 2,7975 & 19.720 & 2.250 \\
\hline Holanda & 5,6781 & 40.020 & 4.570 \\
\hline Irlanda & 1,5814 & 11.140 & 1.270 \\
\hline Italia & 17,7917 & 125.400 & 14.330 \\
\hline Letonia & 0,2746 & 1.930 & 220 \\
\hline 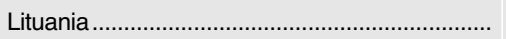 & 0,4063 & 2.860 & 330 \\
\hline 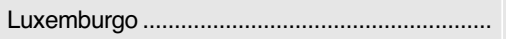 & 0,2487 & 1.750 & 200 \\
\hline 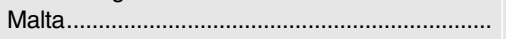 & 0,0726 & 510 & 60 \\
\hline 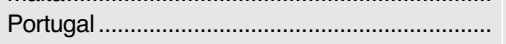 & 2,4921 & 17.560 & 2.010 \\
\hline Total & 100,0000 & 704.800 & 80.550 \\
\hline
\end{tabular}

financiera está compuesta de tres documentos: los Términos Generales, que incluyen las cláusulas estándar de un contrato financiero y son uniformes para todos los contratos de apoyo financiero; los Términos Específicos de cada modalidad de asistencia financiera, que incluyen las cláusulas específicas de cada una de las modalidades de apoyo del ESM y también son uniformes para todos los contratos de apoyo financiero; y el Acuerdo de Asistencia Financiera (FFA, por sus siglas en inglés), que recoge los términos y condiciones financieras (por ejemplo, importe y plazo) de la asistencia financiera, aclara qué Términos Específicos son de aplicación en función del instrumento concedido y determina las condiciones concretas que derogan, modifican o suplementan los Términos Generales y los Términos Específicos.

Esta jerarquía normativa finaliza con las decisiones adoptadas por el MD y por las políticas internas de la institución.

\section{Estructura financiera del ESM}

Inicialmente, el capital autorizado del ESM ascendía a 700.000 millones de euros, que quedaban divididos en siete millones de acciones, cada una con un valor nominal de 100.000 euros. Con las adhesiones de Letonia y Lituania a la zona euro en 2014 y 2015 respectivamente, el capital autorizado del ESM ha pasado a ser de 704.800 millones de euros.

A su vez, el capital autorizado del ESM se divide en capital desembolsado y capital suscrito pero no desembolsado. Inicialmente, el ESM contaba con un capital desembolsado de 80.000 millones de euros, que tras las adhesiones de Letonia y Lituania $^{12}$, ha pasado a ser de 80.550 millones de euros.

La participación de los Estados miembros de la zona euro en el capital del ESM se calcula de acuerdo con la clave de contribución del BCE, que toma en consideración el porcentaje que ese país representa sobre el total en términos de población y de producto interior bruto, otorgando la misma ponderación a ambos criterios.

La Tabla 1 muestra la clave actual de contribución de cada uno de los 19 Estados miembros de la zona euro, así como los importes de capital desembolsado y exigible.

12 Dado que el PIB per cápita de Letonia y Lituania es inferior al 75 por 100 de la media de la Unión Europea, ambos países se benefician de una corrección temporal de 12 años en su contribución de capital, calculada de acuerdo con la fórmula recogida en el artículo 42 del Tratado del ESM. 
El capital suscrito pero no desembolsado podría ser llamado en tres circunstancias. En primer lugar, podría producirse una llamada general de capital en caso de que el BoG tomara esta decisión por unanimidad. Este supuesto podría darse, por ejemplo, en el contexto de un incremento de la capacidad de préstamo del ESM. En segundo lugar, la llamada de capital podría producirse con el fin de reponer el capital desembolsado, bien para mantener la ratio mínima del 15 por 100 entre capital desembolsado y capacidad de préstamo del ESM, bien para hacer frente a las pérdidas procedentes del impago de algún Estado miembro beneficiario. En este segundo caso, la llamada de capital quedaría aprobada con la mayoría simple del Consejo de Administración. En tercer lugar, el Director Ejecutivo del ESM podría proponer una llamada de capital de emergencia para evitar el incumplimiento de obligaciones de pago del ESM. Los Estados miembros dispondrían de siete días para aportar el capital exigido bajo este tercer supuesto.

La capacidad inicial de préstamo del ESM se encontraba en $\mathbf{5 0 0 . 0 0 0}$ millones de euros. En la actualidad, esta capacidad de préstamo se encuentra en 369.310 millones de euros, al haber comprometido 8.968 millones de euros y hasta 86.000 millones de euros bajo los programas de asistencia financiera para Chipre y Grecia, respectivamente, y al existir 35.721 millones de euros pendientes de amortización bajo la asistencia financiera para la recapitalización de las entidades financieras españolas.

El capital desembolsado no se emplea para aportar asistencia financiera a los Estados miembros de la zona euro que la necesiten. De hecho, los fondos correspondientes al capital desembolsado se invierten en activos de la máxima calidad crediticia, en línea con la Política de Inversiones del ESM.

El capital desembolsado permite el apalancamiento del ESM en los mercados financieros y en las entidades de crédito. Son precisamente los fondos captados a través de estas vías los que se emplean para los programas de asistencia financiera de los Estados miembros de la zona euro que así lo requieren. En el orden de prelación de acreedores, el ESM goza del estatus de acreedor preferente por detrás del Fondo Monetario Internacional.

EI ESM se financia en mercados a través de subastas, sindicaciones y colocaciones privadas. Cuenta con una estrategia de financiación diversificada, basada en un pool de financiación a corto plazo y un pool de financiación a medio y largo plazo. En este sentido, el ESM emite tanto instrumentos en los mercados de capitales (bonos con vencimientos entre 1 y 30 años) como en los mercados monetarios (letras). Adopta una estrategia de pooled funding, por lo que las emisiones no van afectas a la asistencia financiera para un determinado Estado miembro.

EI ESM lanzó su programa de financiación a corto plazo el 8 de enero de 2013, con una primera emisión de letras. El programa a medio y largo plazo se lanzó más tarde, el 8 de octubre de 2013, con una emisión de un bono a cinco años por importe de 7.000 millones de euros.

A efectos prudenciales, los bonos del ESM reciben una ponderación por riesgo del 0 por 100 y son clasificados como activos líquidos de nivel 1 en la ratio de cobertura de liquidez.

\section{Procedimiento general de concesión de un programa de asistencia financiera}

Los Estados miembros del ESM podrán dirigir una solicitud de asistencia financiera al Presidente del BoG, indicando el instrumento de asistencia financiera al que optan.

Una vez recibida la solicitud, el Presidente del BoG encomendará a la Comisión Europea, en coordinación con el Banco Central Europeo, la realización de los siguientes análisis: primero, la evaluación de la existencia de un riesgo para la estabilidad financiera del conjunto de la zona euro o de sus Estados miembros; segundo, el análisis de sostenibilidad de la deuda pública, conjuntamente con el Fondo Monetario Internacional cuando fuera $\triangle$ 
adecuado y posible; y tercero, la evaluación de las necesidades reales o potenciales de financiación del Estado miembro solicitante.

Tras la presentación de estos análisis por las instituciones, el BoG puede decidir conceder, en principio, asistencia financiera al Estado miembro solicitante.

A continuación, el BoG encomendará a la Comisión Europea la negociación de un Memorando de Entendimiento (MoU, por sus siglas en inglés) con el Estado miembro solicitante, en el que se definirá con precisión la condicionalidad asociada al instrumento financiero correspondiente. Paralelamente, el MD del ESM preparará una propuesta de FFA.

Posteriormente, el BoG aprobará el MoU propuesto por la Comisión Europea y la propuesta de FFA del ESM. Finalmente, la Comisión Europea firmará el MoU en nombre del ESM y la FFA se someterá a la aprobación del BoD.

Una vez aprobado el programa de asistencia financiera, la Comisión Europea, en coordinación con el Banco Central Europeo y con el Fondo Monetario Internacional, velará por el cumplimiento de la condicionalidad asociada al instrumento de asistencia financiera. Los informes reportados por estas instituciones serán claves a la hora de proceder con los desembolsos al Estado miembro beneficiario.

Asimismo, el ESM ha establecido un sistema de alerta temprana para garantizar que recibe a su debido tiempo el reembolso de las sumas concedidas.

\section{Instrumentos de asistencia financiera}

\subsection{Asistencia financiera precautoria}

El objetivo fundamental de las líneas de crédito precautorias es garantizar el acceso continuado al mercado de Estados miembros con sólidos fundamentos económicos, aislándolos de posibles tensiones financieras.

Las líneas de crédito precautorias son de dos tipos: Línea de Crédito Precautorio Condicionada
(PCCL, por sus siglas en inglés) y Línea de Crédito con Condiciones Reforzadas (ECCL, por sus siglas en inglés).

La PCCL está disponible para aquellos Estados miembros cuya situación económica y financiera sea estable, respetando determinados criterios de elegibilidad, marcados por la sostenibilidad de la deuda pública, el respeto a los compromisos adquiridos bajo el Procedimiento de Déficit Excesivo, una historia de acceso a los mercados de capitales en condiciones razonables, una posición externa sostenible y la ausencia de problemas en el sector bancario que pudieran suponer una amenaza para el conjunto de la zona euro. En caso de solicitar el desembolso de fondos, el Estado miembro será sometido a supervisión reforzada.

La ECCL está disponible para aquellos Estados miembros cuya situación económica y financiera sea estable, pero que no cumplan con alguno de los criterios de elegibilidad exigibles para la aplicación de la PCCL. El Estado miembro que solicite la ECCL deberá adoptar medidas correctivas para hacer frente a las debilidades detectadas y evitar problemas en el futuro acceso a los mercados financieros. Desde el momento de la concesión de la ECCL, y aunque el Estado miembro no haya solicitado el desembolso de fondos, se iniciará el procedimiento de supervisión reforzada.

Hasta la fecha, ninguna de estas modalidades de asistencia financiera precautoria ha sido empleada.

\subsection{Instrumento de recapitalización bancaria indirecta}

Bajo el instrumento de recapitalización bancaria indirecta, un Estado miembro contrae un préstamo con el ESM, cuyos fondos son canalizados por el propio Estado a las correspondientes entidades financieras. Por tanto, se trata de una modalidad de asistencia financiera adecuada para los casos en que las fuentes de inestabilidad financiera o económica proceden del propio sistema financiero y no de la adopción de determinadas políticas fiscales o estructurales. 
Para poder acceder a este instrumento, el Estado miembro solicitante de la asistencia financiera ha de cumplir una serie de requisitos de elegibilidad. En primer lugar, el Estado ha de demostrar la falta de alternativas, tanto privadas como públicas, para recapitalizar las correspondientes entidades financieras. En segundo lugar, las entidades financieras a recapitalizar han de tener relevancia sistémica o suponer una seria amenaza para la estabilidad financiera del conjunto de la zona euro. Finalmente, el Estado miembro solicitante ha de demostrar su capacidad de devolver los fondos prestados, aún en los casos en que no fuera capaz de recuperar el capital inyectado en las entidades beneficiarias de acuerdo con el calendario previsto en las decisiones sobre ayudas de Estado.

Esta fue la modalidad de asistencia financiera concedida al Reino de España en 2012, bajo la que se desembolsaron 41.333 millones de euros en dos desembolsos: el primero, realizado el 11 de diciembre de 2012, tuvo un importe total de 39.468 millones de euros y se destinó a la recapitalización de las entidades de crédito del Grupo 1 y de la Sociedad de Gestión de Activos Procedentes de la Reestructuración Bancaria $\left(\right.$ Sareb) ${ }^{13}$; el segundo, realizado el 5 de febrero de 2013, alcanzó un importe de 1.865 millones de euros y se empleó para la recapitalización de las entidades de crédito del Grupo $2^{14}$.

El programa de asistencia financiera español finalizó en diciembre de 2013. Hasta la fecha, se han realizado tres preamortizaciones voluntarias ${ }^{15}$ y una obligatoria ${ }^{16}$. El principal pendiente, 35.721

\footnotetext{
13 El destino de los fondos fue el siguiente: 17.959 millones de euros para BFA-Bankia, 9.084 millones de euros para CatalunyaCaixa, 5.425 millones de euros para NCG Banco, 4.500 millones de euros para Banco de Valencia y 2.500 millones de euros para Sareb.

14 El destino de los fondos fue el siguiente: 730 millones de euros para Banco Mare Nostrum, 604 millones de euros para Banco Ceiss, 407 millones de euros para Caja 3 y 124 millones de euros para Liberbank.

${ }^{15}$ Las fechas y cantidades de las tres preamortizaciones voluntarias fueron las siguientes: el 8 de julio de 2014, se preamortizaron $1.304 \mathrm{mi}-$ llones de euros; el 17 de marzo de 2015, se preamortizaron 1.500 millones de euros; y el 14 de julio de 2015 , se preamortizaron 2.500 millones de euros.

${ }^{16}$ El 23 de julio de 2014, se devolvieron 308 millones de euros, que no fueron empleados por el FROB para la suscripción de deuda subordinada o capital en Sareb.
}

millones de euros, acabará de devolverse en 2027, si se sigue el calendario de amortizaciones previsto en la actualidad.

\subsection{Préstamos convencionales}

El objetivo de los préstamos convencionales del ESM es asistir a aquellos Estados miembros que presentan elevadas necesidades de financiación pero que han perdido acceso a los mercados financieros, bien porque no encuentran prestamistas 0 porque los prestamistas ofrecen financiación a costes muy elevados. Con el fin de asegurar que el Estado miembro retorna plenamente a los mercados financieros y retoma la senda del crecimiento económico y la consolidación fiscal, los préstamos convencionales del ESM están sometidos a un programa de ajuste macroeconómico completo, preparado por la Comisión Europea conjuntamente con el Banco Central Europeo y el Fondo Monetario Internacional.

Hasta el momento, dos son los Estados miembros que han resultado beneficiarios de un préstamo convencional del ESM, ligado a un programa de ajuste macroeconómico completo: Chipre y Grecia.

Aunque Chipre dirigió una solicitud de asistencia financiera al Presidente del Eurogrupo el 25 de junio de 2012, el BoD no aprobó la FFA chipriota hasta el 8 de mayo de 2013. El ESM se comprometió a prestar asistencia financiera a Chipre hasta un importe de 8.968 millones de euros, que serían complementados por un préstamo de 1.000 millones de euros del Fondo Monetario Internacional. El programa de ajuste macroeconómico chipriota comprende la reestructuración y reducción del tamaño de su sistema financiero, así como medidas de consolidación fiscal y reforma estructural. Está previsto que el programa finalice el 31 de marzo de 2016. Hasta el momento, el ESM ha desembolsado 6.300 millones de euros.

El 8 de julio de 2015, el Gobierno griego presentó una solicitud de asistencia financiera al Presidente del Eurogrupo. Tras las correspondientes $D$ 
negociaciones, el BoG aprobó el MoU griego el 19 de agosto de 2015. En este caso, el MoU contiene medidas a aplicar por las autoridades griegas en cuatro áreas: sostenibilidad fiscal, estabilidad financiera, crecimiento económico, competitividad e inversión y administración pública. El compromiso del ESM con Grecia es de hasta 86.000 millones de euros, aunque se espera que esta cifra se vea reducida con la posterior participación del Fondo Monetario Internacional en el programa. Hasta el momento, Grecia ha recibido un único desembolso bajo este programa, por importe de $23.000 \mathrm{mi}$ llones de euros, de los cuales 10.000 millones de euros han sido desembolsados en bonos del ESM en una cuenta intermedia del ESM, esperando a ser empleados en las operaciones de recapitalización bancaria.

\subsection{Instrumento de apoyo del mercado primario}

Bajo este instrumento, el ESM podrá adquirir, en mercado primario y a precios de mercado, bonos u otro tipo de activos financieros emitidos por el Estado miembro solicitante. El objetivo fundamental de este instrumento es permitir que el Estado miembro beneficiario mantenga o restablezca sus relaciones con la comunidad inversora, reduciendo así los riesgos de una subasta fallida. Para poder activar este instrumento, es necesario que el Estado miembro se encuentre bajo un programa de ajuste macroeconómico completo o que haya solicitado la realización de desembolsos bajo alguna de las facilidades de asistencia financiera precautoria.

Hasta el momento, este instrumento no ha sido empleado.

\subsection{Instrumento de apoyo del mercado secundario}

El ESM podrá intervenir en los mercados secundarios de deuda pública de aquellos Estados miembros solicitantes cuya estabilidad financiera esté en riesgo como consecuencia de la ausencia de liquidez en los mercados financieros. El Estado miembro podrá solicitar la aplicación de este instrumento con independencia de estar sometido o no a un programa de ajuste macroeconómico completo. Si el Estado miembro solicitante no estuviera sometido a un programa de ajuste macroeconómico completo, sólo podrá acceder al instrumento de apoyo del mercado secundario si cumple determinados requisitos de estabilidad financiera ${ }^{17}$, valorados conjuntamente por la Comisión Europea y el Banco Central Europeo.

\subsection{Instrumento de recapitalización bancaria directa}

Las Conclusiones del Consejo Europeo de 14 de diciembre de 2012 acordaron cuáles serían los principales componentes del proyecto de unión bancaria. Además del Mecanismo Único de Supervisión y el Mecanismo Único de Resolución, se aludió a la posibilidad de que el ESM recapitalizara bancos directamente.

El artículo 19 del Tratado del ESM prevé la posibilidad de que el BoG revise la lista de instrumentos de asistencia financiera contemplada en los artículos 14 a 18, introduciendo cambios en ella. Así, el 8 de diciembre de 2014, el BoG, cumpliendo el mandato dado por el Consejo Europeo, aprobó la introducción de un nuevo instrumento: el instrumento de recapitalización bancaria directa (DBRI, por sus siglas en inglés).

Hasta la introducción de este instrumento, el ESM sólo podía recapitalizar entidades de crédito de forma indirecta: el ESM proporcionaba un préstamo al Estado miembro, que a su vez ingresaba $\triangleright$

17 Estos requisitos son los mismos que han de verificarse para conseguir la aplicación de la PCCL, es decir: 1) respeto a las reglas del Pacto de Estabilidad y Crecimiento; 2) deuda pública sostenible; 3) respeto a los compromisos asumidos bajo el Procedimiento de Desequilibrios Macroeconómicos Excesivos; 4) registro de acceso a los mercados internacionales de capitales en condiciones razonables; 5) posición externa sostenible; 6) ausencia de problemas de solvencia en las instituciones financieras del país. 
los fondos en las entidades pertinentes. Como ya se ha explicado, ésta fue la modalidad de asistencia financiera aplicada en el caso español.

No obstante, la modalidad de recapitalización bancaria indirecta implica un incremento en el nivel de deuda pública del Estado miembro que obtiene la asistencia, reforzando el círculo vicioso entre el soberano y las entidades de crédito. Por ello, el DBRI, al permitir que el ESM recapitalice directamente una entidad de crédito sin tener que pasar por el soberano, reduce esta perniciosa conexión.

Desafortunadamente, las condiciones necesarias para la aplicación del DBRI son demasiado restrictivas, lo que reduce enormemente las posibilidades de su activación.

En primer lugar, el DBRI resultará de aplicación sólo a entidades de crédito consideradas de relevancia sistémica o que puedan suponer una clara amenaza a la estabilidad financiera del conjunto de la zona euro.

En segundo lugar, el DBRI será un instrumento de último recurso, antes de cuya aplicación tanto el sector privado como el sector público nacional deberán haber asumido su parte en el rescate de la entidad de crédito y que éste se haya verificado insuficiente.

Desde el punto de vista privado, ha de haberse producido un bail-in de al menos el 8 por 100 de los pasivos totales de la entidad de crédito, una contribución del Fondo Único de Resolución de al menos el 5 por 100 de estos pasivos y la conversión o quita de todas las deudas no aseguradas.

Desde el punto de vista público, ha de verificarse que el Estado miembro donde reside la entidad de crédito en problemas no es capaz de proporcionarle apoyo público sin poner en riesgo su sostenibilidad fiscal. En cualquier caso, el Estado miembro deberá coparticipar junto con el ESM en la recapitalización de la entidad de crédito. Así, en caso de que el capital ordinario de nivel 1 (CET 1) fuera inferior al 4,5 por 100 , el Estado miembro debería reponer el capital de la entidad hasta este nivel. Una vez alcanzado dicho nivel, el Estado miembro debería contribuir con al menos el 20 por 100 de la inyección de fondos públicos durante los dos primeros años desde la entrada en vigor del DBRI y con el 10 por 100 a partir de ese momento.

Por último, el ESM dispondrá únicamente de 60.000 millones de euros para proceder a la recapitalización directa de entidades de crédito en dificultades.

A pesar de que el diseño actual del DBRI admite mejoras, es necesario destacar que la zona euro ha dado un paso muy importante al permitir su introducción. En cualquier caso, la incorporación de posibles mejoras al instrumento no es descartable. En este sentido, cabría hacer referencia al Informe de los Cinco Presidentes, que sugiere la necesidad de revisar el diseño del instrumento en el futuro como mecanismo para completar el proyecto de la unión bancaria.

\section{Conclusiones}

Con la creación del ESM, la zona euro ha dado un gran paso en la profundización de la unión monetaria, sentando las bases de una verdadera cooperación en la prevención y resolución de crisis económicas y financieras. No obstante, la naturaleza del ESM puede seguir evolucionando, ampliando sus funciones y contribuyendo al fortalecimiento del proyecto de moneda única. En este sentido, cabe destacar el Informe de los Cinco Presidentes, en el que se sugiere la posibilidad de que el ESM dote una línea de crédito a favor del Fondo Único de Resolución como mecanismo para garantizar el common backstop. Este mismo informe alude a la posibilidad de que el ESM se integre en el medio plazo (entre julio de 2017 y 2025) en el marco normativo de la Unión Europea. Por otro lado, Jean Claude Juncker, Presidente de la Comisión Europea, aludió en el Discurso del Estado de la Unión Europea, a la posibilidad de emplear el ESM como base para la creación de un Tesoro común de la zona euro.

En conclusión, es innegable que la zona euro ha avanzado notablemente con la creación del $D$ 
ESM. El futuro de esta nueva institución, que podrá ver sus funciones ampliadas, se definirá en los años venideros.

\section{Bibliografía}

[1] FACILIDAD EUROPEA DE ESTABILIZACIÓN FINANCIERA (2015). EFSF Frequently asked questions. Disponible en:

http://www.efsf.europa.eu/attachments/2015-0319\% 20EFSF\%20FAQ.pdf

[2] FACILIDAD EUROPEA DE ESTABILIZACIÓN FINANCIERA (2015). Frequently asked questions on the EFSF financial assistance programme for Ireland. Disponible en:

http://www.efsf.europa.eu/attachments/FAQ\%20Ireland \%20final.pdf

[3] FACILIDAD EUROPEA DE ESTABILIZACIÓN FINANCIERA (2015). Frequently asked questions on the EFSF financial assistance programme for Portugal. Disponible en:

http://www.efsf.europa.eu/attachments/FAQ\%20on\% 20 Portugal\%20exit.pdf

[4] JUNCKER, J.C. (2015). State of the Union 2015. Disponible en:

http://ec.europa.eu/priorities/soteu/docs/state_of_the_ union_2015_en.pdf

[5] JUNCKER, J.C. en coop. con DIJSSELBLOEM, J.; DRAGUI, M.; SCHULZ, M. y TUSK, D. (2015). Completing Europe's Economic and Monetary Union. Disponible en:

http://ec.europa.eu/priorities/economic-monetaryunion/docs/5-presidents-report_en.pdf

[6] MECANISMO EUROPEO DE ESTABILIDAD (2015). Frequently asked question on the European Stability Mechanism. Disponible en: http://www.esm.europa.eu/pdf/2015-09-02\% 20FAQ\%20ESM.pdf

[7] MECANISMO EUROPEO DE ESTABILIDAD (2015). Frequently Asked Questions on ESM paidin capital. Disponible en:

http://www.esm.europa.eu/pdf/FAQ\%20on\%20 paid-in\%20capital\%202015.pdf

[8] MECANISMO EUROPEO DE ESTABILIDAD (2015). Frequently asked questions on the ESM financial assistance programme for Spain and the country's successful exit. Disponible en: http://www.esm.europa.eu/pdf/FAQ\%20Spanish\%20 exit.pdf
[9] MECANISMO EUROPEO DE ESTABILIDAD (2015). Frequently asked questions on the financial assistance for Cyprus. Disponible en:

http://www.esm.europa.eu/pdf/FAQ\%20Cyprus\% 2018092013.pdf

[10] MECANISMO EUROPEO DE ESTABILIDAD (2015). Frequently asked questions on EFSF/ESM financial assistance for Greece. Disponible en: http://www.esm.europa.eu/pdf/2015-08-19\% 20FAQ\%20Greece.pdf

[11] MECANISMO EUROPEO DE ESTABILIDAD (2015). European Stability Mechanism Guideline on Loans. Disponible en:

http://www.esm.europa.eu/pdf/ESM\%20Guideline\% 20on\%20loans.pdf

[12] MECANISMO EUROPEO DE ESTABILIDAD (2015). European Stability Mechanism Guideline on Primary Market Support Facility. Disponible en: http://www.esm.europa.eu/pdf/ESM\%20Guideline\% 20on\%20the\%20primary\%20market\%20support \%20facility.pdf

[13] MECANISMO EUROPEO DE ESTABILIDAD (2015). European Stability Mechanism Guideline on Precautionary Financial Assistance

Disponible en:

http://www.esm.europa.eu/pdf/ESM\%20Guideline\% 20on\%20precautionary\%20financial\%20assistance. pdf

[14] MECANISMO EUROPEO DE ESTABILIDAD (2015). European Stability Mechanism Guideline on Precautionary Financial Assistance. Disponible en: http://www.esm.europa.eu/pdf/ESM\%20Guideline\% 20on\%20precautionary\%20financial\%20assistance. pdf

[15] MECANISMO EUROPEO DE ESTABILIDAD (2015). European Stability Mechanism Guideline on Recapitalisation of Financial Institutions. Disponible en:

http://www.esm.europa.eu/pdf/ESM\%20Guideline\%20 on $\% 20$ recapitalisation $\% 20$ of\%20financial\%20 institutions_with\%20notice.pdf

[16] MECANISMO EUROPEO DE ESTABILIDAD (2015). European Stability Mechanism Guideline on Financial Assistance for the Direct Recapitalisation of Institutions. Disponible en:

http://www.esm.europa.eu/pdf/20141208\%20Guideline \%20on\%20Financial\%20Assistance\%20for\%20the \%20Direct\%20Recapitalisation\%20of\%20Institutions. pdf

[17] UNIÓN EUROPEA. Reglamento (UE) 2015/1360 del Consejo, de 4 de agosto de 2015, que modifica el Reglamento (UE) $n^{\circ} 407 / 2010$ por el que se establece un mecanismo europeo de estabilización financiera. 